Hadron Structure '15

International Journal of Modern Physics: Conference Series

Vol. 39 (2015) 1560099 (6 pages)

(C) The Author(s)

DOI: 10.1142/S201019451560099X

\title{
Simulation of the SCAN3 setup
}

\author{
S. V. Afanasev, B. V. Dubinchik, D. K. Dryablov* and Z. A. Igamkulov \\ Veksler and Baldin Laboratory of High Energy Physics \\ Joint Institute for Nuclear Research \\ 141980, Dubna, Moscow region, Russia \\ *dryablov@lhe.jinr.ru \\ S. Vokal \\ Faculty of Science, University of P.J. Shafarik \\ Koshice, Slovak Republic \\ stanislav.vokal@upjs.sk
}

Published 26 November 2015

The first results of the geometry GEANT3 simulation of the three-arm magnetic spectrometer SCAN3 are presented in this article. The possibility of detection of exotic nuclei (such as eta-mesic nucleus) with help of the SCAN3 and the Nuclotron internal target is shown.

Keywords: eta-mesic nuclei; Monte Carlo simulation; experiment; exotic nuclei.

PACS Numbers: 07.05.Tp, 21.85.+d

\section{Introduction}

The new project was initialized in the Veksler and Baldin Laboratory of high energy physics of the Joint institute for nuclear research (Dubna, Russia). The project is directed on creation of a three-arms hybrid magnetic spectrometer SCAN3 for research of excited baryonic matter at the internal beam of the Nuclotron accelaretor. The spectrometer is intended for registration and analysis charged particles $(\pi$, $\mathrm{K}, \mathrm{p})$ and neutral particles (n). The spectrometer is designed for research of the pairs of the particles emitted from an interaction point at an angle close to 180 degrees ( so-called wide angular pair correlations). The possibility of registering of such pairs allows the study of a wide range of physical phenomena:

- $\eta$-mesic nucleus, i.e., a quasibound state of the $\eta$-meson and a nucleus (for example, see Ref. 1),

This is an Open Access article published by World Scientific Publishing Company. It is distributed under the terms of the Creative Commons Attribution 3.0 (CC-BY) License. Further distribution of this work is permitted, provided the original work is properly cited. 
- $\Delta$-isobar produced in the target nucleus,

- superdense cold baryonic component,

- correlation of nn and np pairs.

\section{Previous Experimental Setup}

In 2006-2010 a set of experimental data at two-arms nonmagnetic spectrometer SCAN2 and the internal d-beam of the Nuclotron (LHEP, JINR) had been obtained. Due to presence of a strong near-threshold nucleon resonance $S_{11}(1535)$ in the $\eta \mathrm{N}$ channel slow $\eta$-mesons have a strong attraction with nucleons. As a result the slow $\eta$-meson is captured by the nucleus and forms $\eta$-meson nucleus, which in turn breaks up on $\pi \mathrm{N}$ or NN-pairs. The peaks in the invariant mass spectra of back-to-back $\pi \mathrm{p}$ pairs was observed (see Refs. 2 and 3 for more details).

New project provides a significant increase of the energy resolution of the setup by including a magnetic spectrometer in the experimental installation and by increasing the time-of-flight base.

\section{New Experimental SCAN3 Setup}

A schematic view of the spectrometer SCAN3 is presented in Fig. 1. The new setup is planned as three arm spectrometer. The one arm (M-arm) is been constructing as a magnetic arm for charged particles and another two are time-of-flight spectrometers for registration of the neutrons and protons. This combination of detectors can register $\pi \mathrm{n}, \pi \mathrm{p}$, pn and pp pairs. All arms are located in one plane with

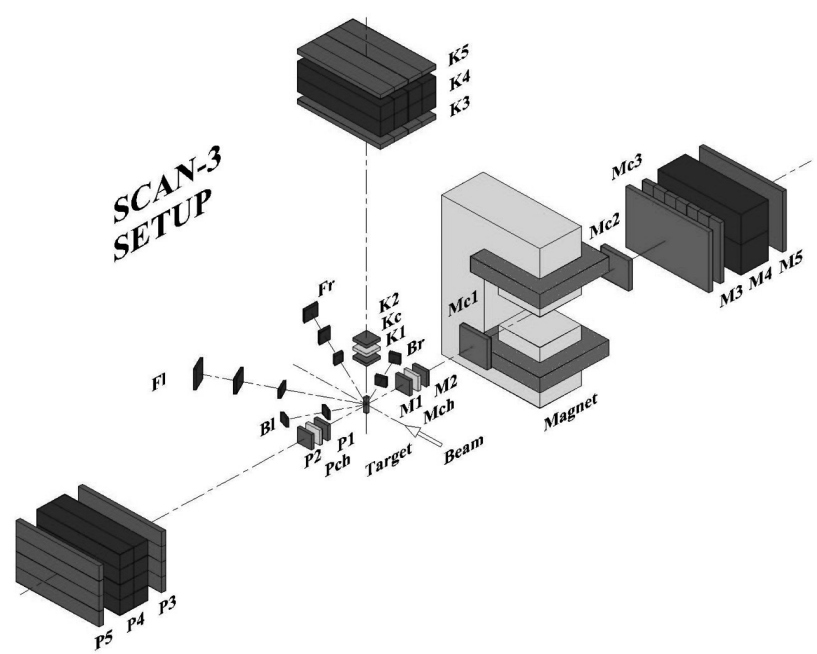

Fig. 1. The experimental SCAN3 setup. Mc1, Mc2 - biplanar drift chambers for precise measurement of the coordinates; Mc3 - multiwire proportional chamber; P1, P2, P3, K1, K2, K3, M1, M2, M3 - trigger time-of-flight scintillation detectors of $\mathrm{P}, \mathrm{K}$ and $\mathrm{M}$ arms; P4, K4 - 2 sets of 8 scintillation detectors, which are used for the registration and spectrometry of neutrons. 
the internal target of the Nuclotron. Two are horizontal and one is vertical. This disposition will be used for determination the studied processes and the background simultaneously.

\section{Requirements to Precisions}

One of the main tasks of the experiment is the allocation and measurement of the narrow peak in the energy distribution of pairs, which are products of $\eta$-meson nucleus decay or $\Delta$-isobar nucleus decay. Based on experimental and theoretical studies (for example, see Refs. 4 and 5) we can formulate the criteria for the required energy resolution of pair. In the case of $\eta$-mesic nucleus decay the required energy resolution is $\delta E=\delta\left(E_{1}+E_{2}\right) \leq 10 \mathrm{MeV}$.

If we consider the $\eta$-meson nucleus decay into $\pi \mathrm{N}$ pair with initial particles at rest, the kinetic energy and momentum of the secondary particles must be equal (if we ignore the effect of binding and Fermi motion) $\mathrm{E}_{\pi}=313 \mathrm{MeV}, \mathrm{E}_{N}=94 \mathrm{MeV}$, $\mathrm{p}_{\pi}=\mathrm{p}_{N}=431 \mathrm{MeV} / \mathrm{c}$. Accordingly, experiment performance demands precisions of measurement of kinetic energy at level of $1 \%$ for pions and $3 \%$ for nucleons.

For the process of the $\eta$-meson nucleus decay into NN pair, the kinetic energy and momentum of the secondary particles must be equal $\mathrm{E}_{N 1}=\mathrm{E}_{N 2}=273 \mathrm{MeV}$ and $\mathrm{p}_{N 1}=\mathrm{p}_{N 2}=767 \mathrm{MeV} / \mathrm{c}$. Accordingly, experiment performance at the declared level demands accuracy in measuring of kinetic energies of such nucleons at level of $1 \%$.

The experimental setup allows to register $\pi \mathrm{n}$ and NN pairs, which are products of $\Delta$-isobar nucleus decay. For these processes, the kinetic energy and momentum of the secondary particles must be equal (if we ignore the effect of binding, Fermi motion and final state interaction) $\mathrm{E}_{n}=26.8 \mathrm{MeV}, \mathrm{E}_{\pi}=126 \mathrm{MeV}, \mathrm{p}_{n}=\mathrm{p}_{\pi}=$ $226 \mathrm{MeV} / \mathrm{c}, \mathrm{E}_{N 1}=\mathrm{E}_{N 2}=146.5 \mathrm{MeV}, \mathrm{p}_{N 1}=\mathrm{p}_{N 2}=544 \mathrm{MeV} / \mathrm{c}$.

\section{Monte Carlo Simulation}

Simulation of the SCAN3 setup with help of the GEANT3 software have been done.

\subsection{Accuracy of determining of the deflection angle in a magnetic field}

We have analyzed the accuracy of determining of the deflection angle of the pions in the magnetic field $(\mathrm{B}=7 \mathrm{kG})$ depending on the thickness of Cherenkov detector Mch and second scintillation counter M2, adding the fourth drift chamber, which is located between the target and a magnet for more accurate determination of the angle, the use of the vacuum instead of the air environment. The kinetic energy of the pions was equal $\mathrm{E}_{\pi}=313 \mathrm{MeV}$, it corresponds to the $\eta$-meson nucleus decay into $\pi \mathrm{N}$ pair ( $\pi$ is registered with the M-arm of the SCAN3, $\mathrm{N}$ is registered with the P-arm of the SCAN3). 
Data analysis showed the following main results for pions in the magnetic field:

(i) the best resolution of the angle have been obtained for the case of the vacuum environment, thin $(0.3 \mathrm{~mm})$ counters Mch and M2, the presence of 4 coordinate drift chambers $-0.82 \%$ (it corresponds to the pion kinetic energy of $2.6 \mathrm{MeV}$, see left part of the Fig. 2),

(ii) the worst angle resolution have been obtained for the case of the air environment, thick $(3 \mathrm{~mm})$ counters Mch and M2, the presence of 3 coordinate drift chambers $-1.4 \%$ (it corresponds to the pion kinetic energy of $4.4 \mathrm{MeV}$, see right part of the Fig. 2) .

For the same conditions, we have simulated the deflection angle of the protons in the magnetic field. The kinetic energy of the protons was equal $\mathrm{E}_{p}=273 \mathrm{MeV}$, it corresponds to the $\eta$-meson nucleus decay into NN pair. Some of important results were:

(i) the angle resolution is equal $1.17 \%$ (it corresponds to the proton kinetic energy of $3.2 \mathrm{MeV}$ ) for the case of the air environment, thin counters Mch and M2, the presence of 4 coordinate drift chambers,
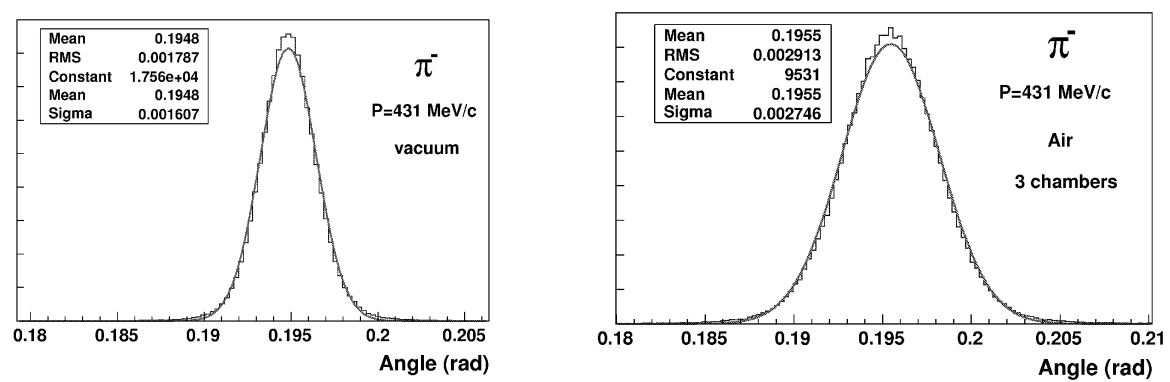

Fig. 2. The distribution of the deflection angle of the pions $\left(\mathrm{E}_{\pi}=313 \mathrm{MeV}\right)$ in the magnetic field $(B=7 \mathrm{kG}$ ) for two different geometries of the SCAN3.
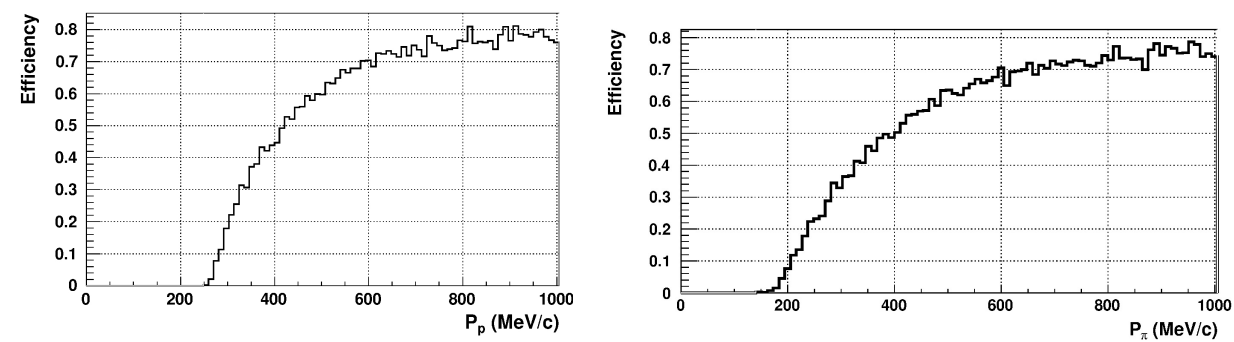

Fig. 3. Momentum efficiency of protons (left) and pions (right) in the M-arm of the SCAN3. 
(ii) the angle resolution is equal $2.4 \%$ (it corresponds to the proton kinetic energy of $6.5 \mathrm{MeV}$ ) for the case of the air environment, thin counters Mch and M2, the presence of 3 coordinate drift chambers.

\subsection{Momentum efficiency}

To calculate the momentum efficiency we have done the following steps. We have simulated protons and pions with uniform momentum distribution from 0 to $1000 \mathrm{MeV} / \mathrm{c}$. We selected particles, which moved from the target in the direction of the poles of the magnet. Of these particles, we chose those that had passed through the magnetic field between the magnet poles and reached scintillation detector M3 (condition for data recording is the presence of signals from the detectors M1, M2 and M3). The Fig. 3 shows the normalized momentum distributions of protons and pions.

\subsection{Effective size of the detectors}

To determine the effective size of the detectors, we have simulated the protons, neutrons, pions with momenta from 0 to $1000 \mathrm{MeV} / \mathrm{c}$. In the case of M-arm we chose pions which have passed through M1, M2, the field between the poles of a magnet and reached the scintillation detector M3. For example, Figs. 4 and 5 show the distribution of the coordinates in the scintillation detector M1 and in the proportional chamber Mc3.
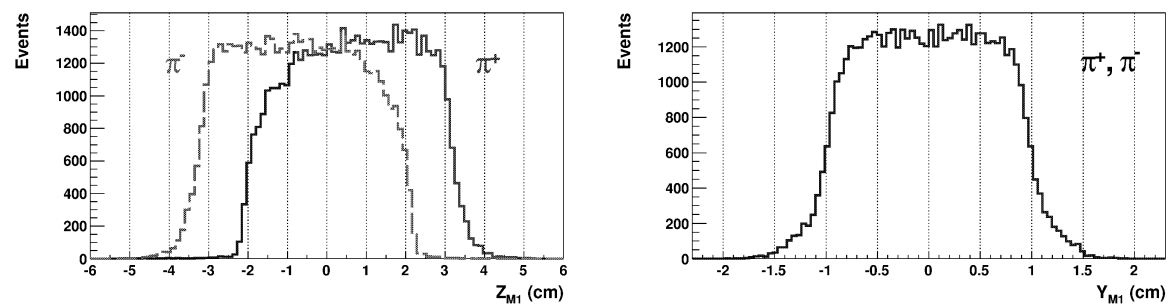

Fig. 4. The distribution of Z-coordinates (left) and Y-coordinates (right) of the pion in the M1.
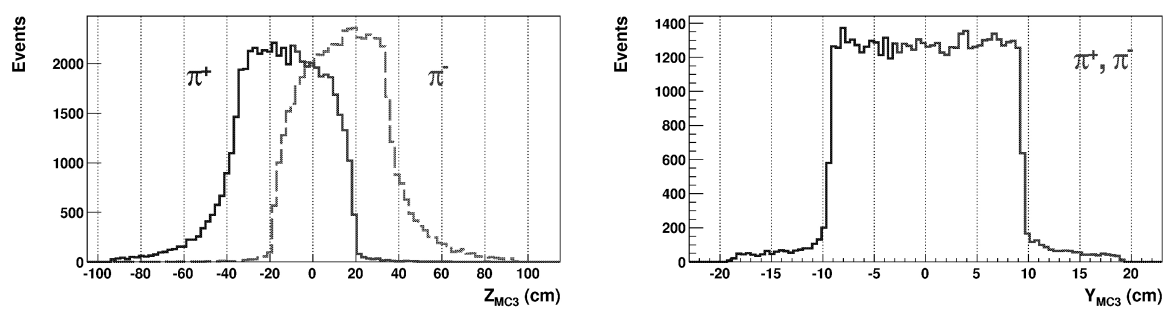

Fig. 5. The distribution of Z-coordinates(left) and Y-coordinates(right) of the pion in the Mc3. 

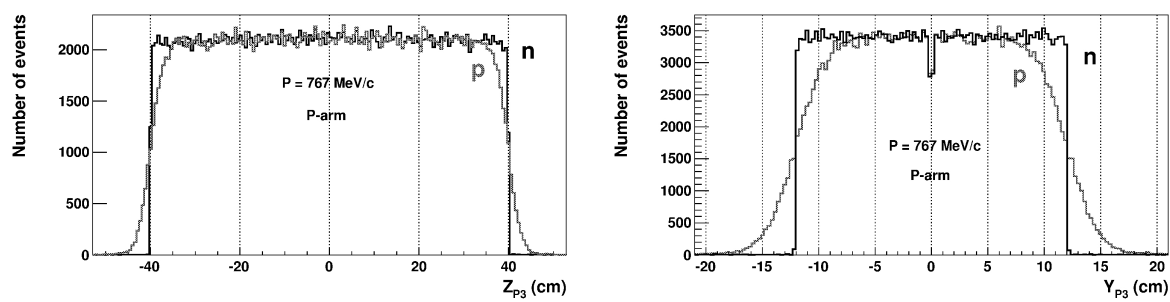

Fig. 6. The distribution of Z-coordinates(left) and Y-coordinates(right) of the proton and neutron in the P3.

The Fig. 6 shows the distribution of the coordinates in a scintillation counter P3 (P-arm) for protons and neutrons, which took off from the target at such angles that the oppositely directed particle fly towards the gap of the magnet (M-arm).

\section{Summary}

Simulation of the three-arm magnetic spectrometer SCAN3 with help of the GEANT3 software have been done.

Using simulated data, the optimum geometry of the SCAN3 setup have been found. The experimental setup can detect pairs of particles $(\pi \mathrm{N}, \mathrm{NN})$, which are products of $\eta$-meson nucleus decay and $\Delta$-isobar nucleus decay.

The energy resolution $\delta E \leq 10 \mathrm{MeV}$ is a condition for the possibility of registration of the $\eta$-meson nucleus. Research on the previous SCAN2 setup showed good energy resolution for protons $(\delta E \approx 5 \mathrm{MeV})$. Simulation of the magnetic arm of the new SCAN3 setup showed that the energy resolution may be approximately equal $1.4 \%$ (for pions) and $2.4 \%$ (for protons). These data provide the energy resolution $\delta\left(E_{1}+E_{2}\right) \leq 10 \mathrm{MeV}$ of $\pi \mathrm{N}$ and $\mathrm{NN}$ pair, which are products of $\eta$-meson nucleus decay.

\section{References}

1. N. G. Kelkar, K. P. Khemchandani, N. J. Upadhyay and B. K. Jain, Rep. Prog. Phys. 76 (2013) 066301.

2. S. V. Afanasev et al., Nuclear Physics B (Proc. Suppl.) 219-220 (2011) 255-258.

3. S. V. Afanasev et al., Nuclear Physics B 245 (2013) 173-176.

4. A. I. L'vov, arXiv:nucl-th/9809054v1, 17 Sep. 1998.

5. M. Pfeiffer et al., Phys. Rev. Lett. 92 (2004) 252001. 